\title{
REFUERZO DE LA ESCAYOLA MEDIANTE FIBRAS DE LANA MINERAL PROCEDENTES DEL RECICLAJE DE RCD
}

\author{
${ }^{1}$ Romaniega Piñeiro, S.; Del Río Merino, M.; Pérez García, C.; San Antonio González, A. \\ ${ }^{1}$ Universidad Politécnica de Madrid. Escuela Universitaria de Arquitectura Técnica. \\ Departamento de Construcciones Arquitectónicas y su control. \\ Avda. Juan de Herrera, 6. 28040 Madrid

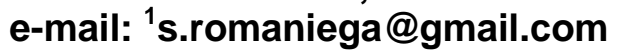

\begin{abstract}
RESUMEN
Con la entrada en vigor del Código Técnico de la edificación el uso de lanas minerales está cada día más generalizado tanto en obras de rehabilitación como en obra nueva. Por lo tanto, la generación de residuos de este tipo de material aislante cobra una mayor importancia.

El objetivo principal de la investigación que se presenta en esta ponencia es analizar la viabilidad del reciclaje de fibras obtenidas de las lanas minerales procedentes de los RCD como material alternativo a las fibras de vidrio cortadas que se utilizan en la actualidad como elementos de refuerzo en los prefabricados de yeso.
\end{abstract}

Para ello se realizan series de escayola E-35 aditivadas con residuo de lana de roca y con residuo de lana de vidrio en diferentes porcentajes de adición. Dichas series se repiten cambiando este aditivo por fibra de vidrio $E$ de $25 \mathrm{~mm}$ de longitud con el fin de realizar un análisis comparativo con respecto a las series aditivadas con residuo de lanas minerales. Todas las series se someten al ensayo Shore C para determinar su dureza superficial y a ensayos mecánicos correspondientes para determinar su resistencia a flexotracción y a compresión.

A partir de los resultados obtenidos se concluye que, con residuo de lana de roca se aumenta la Dureza Shore $\mathrm{C}$ en todos los porcentajes de adición, llegando a mejorar en un $12 \%$ con respecto a la fibra de vidrio, con un porcentaje de adición del $2 \%$. Sin embargo, se obtienen valores inferiores de resistencia a flexotracción para todos sus porcentajes de adición. La mayor diferencia, un 50\%, aparece con el porcentaje de adición del $4 \%$. En el ensayo de resistencia a compresión los resultados obtenidos en las series aditivadas con residuo de lana de roca son superiores para todos los porcentajes de adición estudiados. La mayor diferencia aparece para el $3 \%$ de adición, en el que las series aditivadas con residuo de lana de roca superan en un $36 \%$ a las aditivadas con fibra de vidrio.

Con respecto a las series aditivadas con residuo de lana de vidrio se concluye que, mejoran la dureza superficial linealmente en torno al $5 \%$ para todos los porcentajes de adición, con respecto a las series aditivadas con fibra de vidrio. Los valores de resistencia a flexotracción son superiores hasta el 3\% de adición, llegando esta mejora al $26 \%$ con el $1 \%$ de adición. Los valores obtenidos en el ensayo de resistencia a compresión son mayores para todos los porcentajes de adición, excepto para el $4 \%$. Esta mejora alcanza el $41 \%$ para el porcentaje de adición del 3\%.

Como conclusión final decir que las series aditivadas con lanas minerales procedentes del reciclaje obtienen mejores resultados en los ensayos realizados que las fibras utilizadas en la actualidad como refuerzo del yeso, por lo que resulta viable su sustitución.

Keywords: lana mineral, reciclaje, RCD, escayola, fibras. 


\section{1.- Introducción.}

Durante la última década la intensa actividad en el ámbito de la construcción ha generado grandes cantidades de residuos procedentes de la construcción y demolición (RCD). En Europa se han generado alrededor de 890 millones de toneladas de RCD de media al año, sin embargo, sólo el 50\% de estos RCD generados se reciclan [1].

Debido a la importancia de estos residuos, los países europeos están poniendo en práctica políticas nacionales e internacionales, así como diferentes medidas cuyo objeto es reducir al mínimo los efectos negativos de la generación y la gestión de los residuos para la salud humana y el medio ambiente. La política en materia de residuos tiene también por objeto reducir el uso de recursos y por tanto el impacto medioambiental derivado de la producción de los mismos.

En España se han generado en los últimos años 40 millones de Toneladas de residuos de construcción y demolición. Dentro de estos RCD el $72 \%$ pertenecen a obras residenciales y el $28 \%$ a infraestructuras [2].

Por lo tanto, el sector de la construcción, y en particular la construcción de ámbito residencial, debe asumir el objetivo de reducir el impacto perjudicial que produce, por lo que resulta indispensable introducir nuevas medidas para prevenir la generación de estos residuos o encontrar nuevas vías para su reciclaje.

En España el documento que regula actualmente los residuos de construcción y demolición a nivel nacional es el Real Decreto 105/2008, de 1 de febrero, por el que se regula la producción y gestión de los RCD [3]. De este Real Decreto se derivan los siguientes objetivos:

- Inclusión en los proyectos de obra de un estudio de gestión de RCD.

- Separación en origen de los RCD peligrosos generados en obra y gestión de acuerdo a la legislación de residuos.

- Separación en planta de tratamiento de los residuos peligrosos contenidos en los RCD recibidos y gestión de acuerdo a la legislación de residuos.

- Separación de los RCD en obra, por materiales, a partir de los umbrales establecidos en el Real Decreto 105/2008.

- Cumplimiento del artículo 13 del Real Decreto 105/2008, en cuanto a la utilización (valorización) de residuos inertes procedentes de actividades de construcción y demolición.

- Erradicación del vertido incontrolado de RCD. Desde el 16 de julio de 2009 todos los vertederos en operación en España deben cumplir con los requisitos que les sea de aplicación del Real Decreto 1481/2001.

- Tratamiento de los RCD por gestor autorizado en los términos establecidos en la legislación.

Este Real Decreto se instituye como pieza fundamental de la política española sobre RCD y se espera que contribuya al desarrollo sostenible de un sector tan importante para la economía española como es el sector de la construcción. 


\section{1.- Fibras minerales utilizadas en construcción.}

Los materiales textiles se pueden clasificar según su naturaleza en tres grandes grupos, naturales, artificiales o sintéticos (Tabla 1). En los tejidos naturales las fibras que lo forman se sitúan de manera caótica e irregular por lo que pierden resistencia. Sin embargo, en los tejidos artificiales o en los sintéticos se pueden alinear y juntar al máximo las moléculas de fibra, mejorando su durabilidad y cohesión.

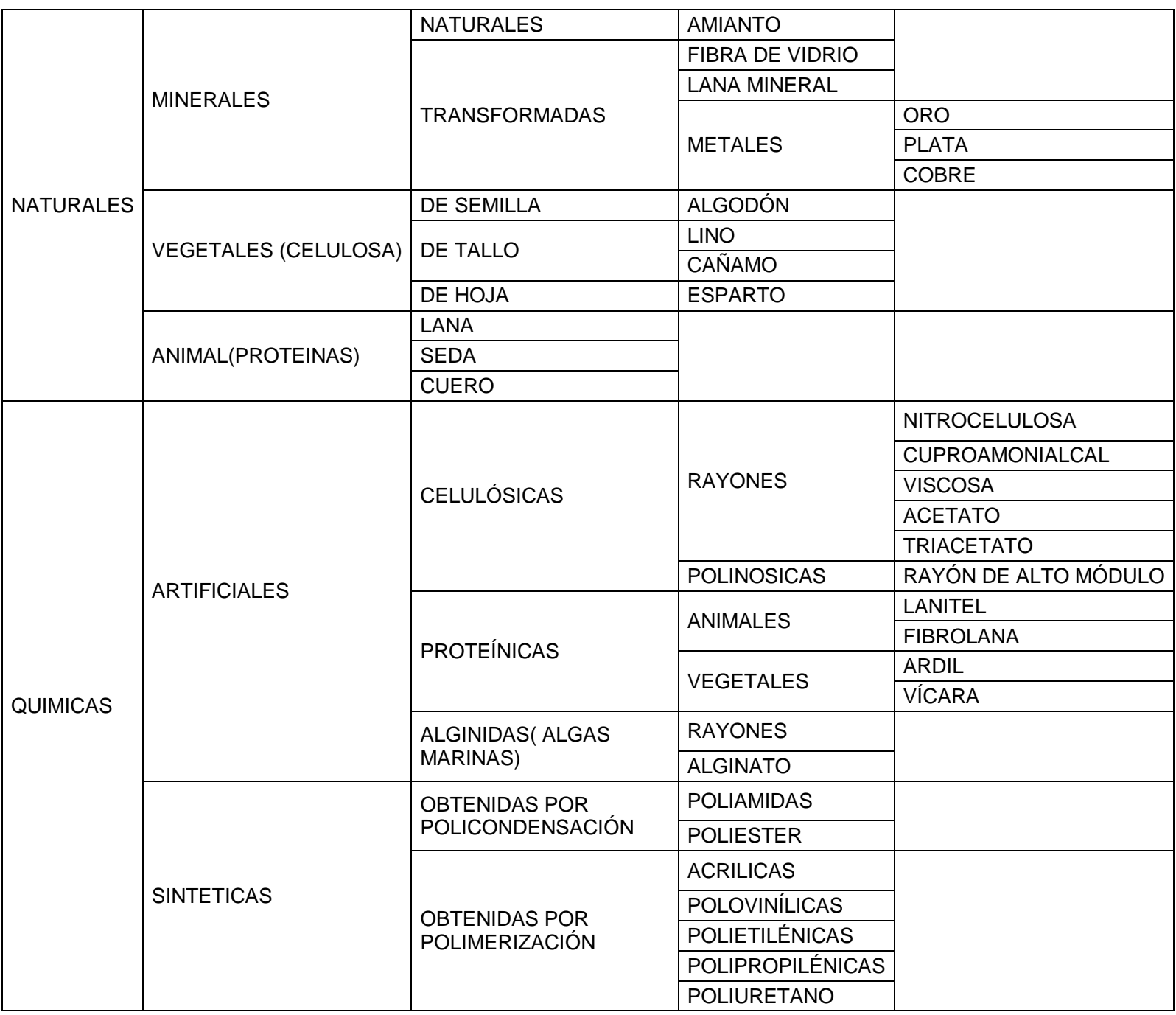

Tabla. 1 "Clasificación de fibras en función de su naturaleza"

Las Fibras Minerales pueden ser naturales, como el Amianto o provenir de materiales hilables como el vidrio o algunos metales. A partir de estas fibras minerales se constituyen las denominadas lanas minerales.

La lana mineral es un material flexible compuesto de fibras inorgánicas constituido por un entrelazado de filamentos de materiales pétreos que forman un fieltro que contiene y mantiene el aire en estado inmóvil. Se obtienen mediante fundición, centrifugación y otros tratamientos y se utilizan en construcción como aislante acústico y térmico. Este material se diferencia de otros aislantes en que es un material resistente al fuego, con un punto de fusión superior a los $1.200^{\circ} \mathrm{C}$.

Existen dos tipos de lanas en función del mineral utilizado como materia prima, la lana de vidrio obtenida a partir de vidrio, y la lana de roca obtenida a partir de roca 
basáltica. Ambas lanas se comercializan en multitud de formatos, principalmente aparecen en forma de panel o lámina rígida o semirrígida, ligados con diferentes tipos de resinas como resinas fenólicas, ignifugas o termoendurecibles, con o sin recubrimiento. Estos recubrimientos pueden llegar a ser muy diferentes, algunos de los más utilizados son: lámina de aluminio, lámina elastómera, velo de poliéster, papel kraft de polietileno, placa de yeso laminado, capa betún oxiasfáltica, capa de polímero intumescente, etc. También se puede encontrar suministrada en forma de rollo o a granel.

Debido a que la lana de roca está fabricada a partir de basalto algunos fabricantes como Rockwooll afirman que es por ello un producto natural $100 \%$ reciclable, y por tanto ideal en el desarrollo de proyectos de construcción sostenible [4]. Por otra parte, otros fabricantes como Isover afirman que las lanas minerales se pueden utilizar para crear nuevas lanas, en concreto nos encontramos con los siguientes porcentajes de reciclado según dicho fabricante, $66 \%$ de lana de roca sobrante de producción y un $75 \%$ en lana de vidrio [5]. También incorpora vidrio reciclado en el proceso de fabricación de la lana de vidrio.

Sin embargo, ambas lanas minerales necesitan grandes cantidades de energía para su fabricación por lo que resulta interesante buscar otro destino tanto para estos sobrantes de fábrica como para los RCD ya que éstos últimos no se someten a ningún tipo de proceso de reciclaje, reutilización o valorización.

Dentro de las fibras minerales utilizadas en construcción se encuentra la fibra de vidrio. La Fibra de Vidrio se obtiene fácilmente del vidrio, calentándolo a la llama y estirándolo con unas pinzas metálicas.

Ésta fibra se comercializa en bruto en forma de hilos de diferentes longitudes para refuerzo de hormigones, morteros o yesos. También se comercializa como material compuesto en multitud de formatos como, tejas acústicas, placas de cartón-yeso reforzadas o láminas asfálticas reforzadas.

\section{Existen cinco grupos:}

-Tipo E: es el tipo de fibra más empleado, se caracteriza por sus propiedades dieléctricas, representa el $90 \%$ de refuerzo para composites.

- Tipo R: se caracteriza porque tiene muy buenas prestaciones mecánicas, demandándose en los sectores de aviación, espacial y armamento.

-Tipo D: su principal característica es su excelente poder dieléctrico, de ello su aplicación en radares, ventanas electromagnéticas...

-Tipo AR: posee un alto contenido en óxido de circonio, el cuál le confiere una buena resistencia a los álcalis.

-Tipo C: se caracteriza por su alta resistencia a agentes químicos.

\section{2.- Antecedentes.}

Las fibras se utilizan desde la antigüedad para reforzar materiales frágiles como el tapial $o$ el adobe. En general, el refuerzo con fibras actúa mejorando el comportamiento físico-mecánico de la matriz. El comportamiento de estos compuestos depende, en primer lugar, del tipo de fibra añadida, y luego de otros factores, como: porcentaje de fibra, longitud de la fibra, orientación, superficie de la fibra, etc. 
Se han encontrado varias referencias bibliográficas sobre la adición de fibras naturales en el refuerzo del yeso/ escayola [6] [7] [8], ninguna de ellas reciclada. Muy utilizadas son las fibras cortas de celulosa, sisal y esparto, pero, de todas ellas, con la que se obtuvieron mejores resultados, fue con la fibra de sisal [9].

En cuanto a la adición de fibras sintéticas y minerales en una matriz de yeso o escayola, se han encontrado numerosas referencias sobre la adición de fibras poliméricas [10] y fibras de vidrio [11] [12], ninguna de ellas procedía del reciclaje. Estas fibras son las más adecuadas ya que el resto son excesivamente caras y de prestaciones mecánicas muy superiores al yeso/escayola, con lo cual estarían infraaprovechadas. Por otro lado, las fibras de vidrio son las fibras que más se utilizan como refuerzo en los prefabricados de escayola.

Existen numerosas investigaciones sobre la incorporación de áridos reciclados en construcción. Estos áridos se agregan en hormigones, morteros y asfaltos sustituyendo a los áridos naturales, con aplicaciones en: bases y subbases de firmes y de carreteras fundamentalmente [13] [14].

También se han encontrado investigaciones sobre el reciclaje de residuos de poliestireno expandido [15] o diversos residuos industriales [16] [17], incorporándolos como cargas en conglomerantes.

Uno de los residuos industriales con aplicaciones en el campo de la construcción es el caucho procedente de neumáticos fuera de uso. Se han encontrado diversas referencias documentales sobre proyectos de investigación que utilizan el granulado de caucho como otro componente más en la fabricación de hormigones, sustituyendo parcialmente a los áridos finos o a las gravas [18].

Se considera que aunque se han encontrado referencias bibliográficas sobre la adición de RCD en una matriz de escayola, como el corcho reciclado [19], no se han encontrado referencias sobre la utilización de fibras minerales procedentes del reciclaje en conglomerados, en concreto en una matriz de escayola.

\section{3.- Objetivo.}

El objetivo principal de este estudio es analizar la viabilidad del reciclaje de fibras obtenidas a partir de las lanas minerales procedentes de los RCD como material alternativo a las fibras de vidrio cortadas que se utilizan en la actualidad como elementos de refuerzo en los prefabricados de yeso.

\section{4.- Método experimental.}

Se confeccionan diferentes probetas de dimensiones $4 \times 4 \times 16 \mathrm{~cm}$ utilizando los siguientes materiales: escayola E-35, lana de roca procedente del reciclaje, lana de vidrio procedente del reciclaje y fibra de vidrio $E$ de $25 \mathrm{~mm}$.

En una primera fase se realizan como referencia series de escayola E-35 y relación A/E 0,6 aditivadas con fibra de vidrio $E$ de $25 \mathrm{~mm}$ en los siguientes porcentajes de adición; $1 \%, 1,5 \%, 2 \%, 2,5 \%, 3 \%, 3,5 \%$ y $4 \%$. 
Como segunda fase se realizan series de escayola E-35 y relación A/E 0,6 aditivadas con lana de roca procedente del reciclaje y lana de vidrio procedente del reciclaje, ambas en formato polvo, en los siguientes porcentajes de adición; $1 \%$, $1,5 \%, 2 \%, 2,5 \%, 3 \%, 3,5 \%$ y $4 \%$. Estas series se confeccionan con las lanas minerales en formato polvo ya que al tratarse de mantas recicladas aglomeradas con resinas no resulta viable su incorporación en formato fibra.

Sobre estas series se realizan ensayos mecánicos para determinar la resistencia a flexión y compresión de las probetas, y el ensayo Shore C para determinar la dureza superficial de las mismas. Todo ello según norma UNE EN 13279-2.

\section{5.- Resultados y discusión.}

Se analizan los diferentes comportamientos en cuanto a dureza superficial, resistencia a flexotracción y resistencia a compresión de la escayola aditivada con lana mineral procedente del reciclaje, comparando estos resultados con los obtenidos para las probetas de escayola aditivada con fibra de vidrio E de $25 \mathrm{~mm}$, según los diferentes porcentajes de adición.

\section{1.- Dureza shore C.}

Destaca que para todos los porcentajes de adición se obtienen unos valores mayores de dureza superficial en las series realizadas con lana de roca procedente del reciclaje. La mayor diferencia la encontramos para un porcentaje de adición del $2 \%$, en este punto las series aditivadas con lana de roca superan en un $14 \%$ a las aditivadas con fibra de vidrio. Para los porcentajes de adición del $1 \%$ y $1,5 \%$ la diferencia se sitúa en el 12\%. A partir del 2,5\% de adición los valores se igualan manteniéndose muy similares hasta el $4 \%$ de adición estudiado (fig. 1).

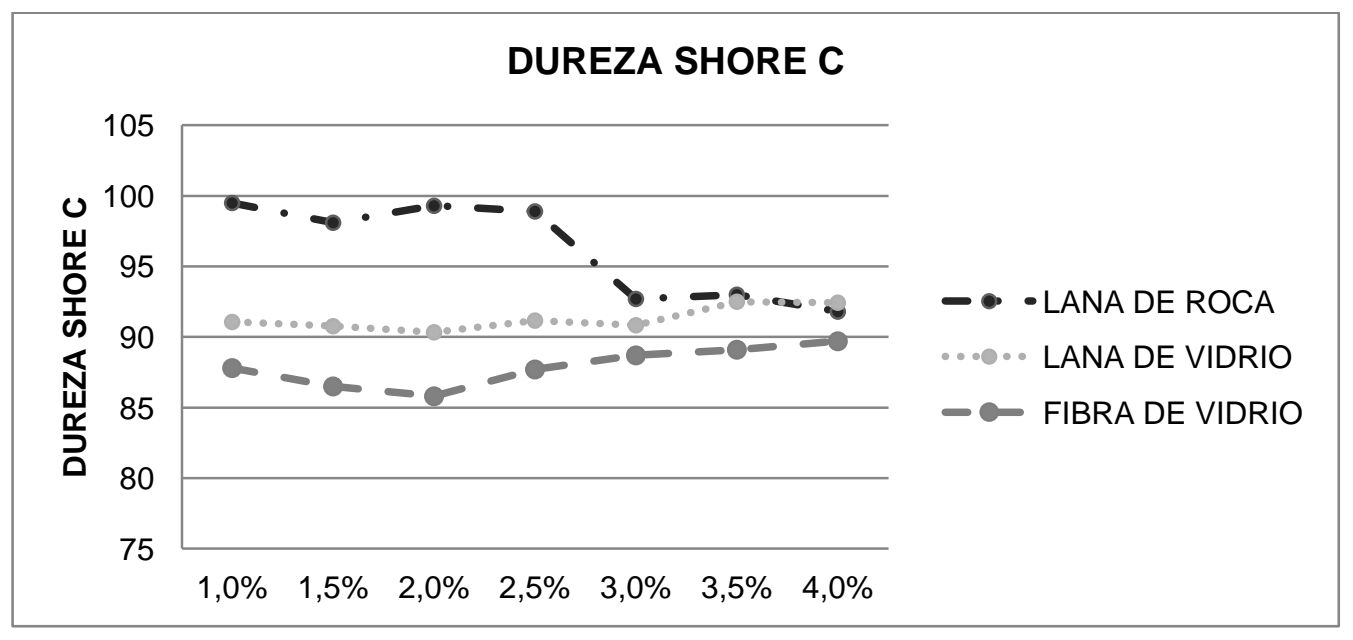

fig. 1 "Comparativa Dureza Shore C"

Las series aditivadas con lana de vidrio obtienen también valores superiores a las aditivadas con fibra de vidrio para todos los porcentajes de adición estudiados. En este caso los resultados son muy similares, apareciendo la mayor diferencia, un $5 \%$, para los porcentajes de adición del $1,5 \%$ y $2 \%$. 


\section{2.- Resistencia a flexotracción.}

Las probetas aditivadas con lana de roca obtienen unos valores inferiores para todos sus porcentajes de adición que las aditivadas con fibra de vidrio. Los valores van en aumento alcanzando su máximo para el 2,5\% de adición, en este punto la diferencia con respecto a las series aditivadas con fibra de vidrio es de un $16 \%$ (fig. 2). El valor más cercano entre estas dos adiciones se encuentra en el $1 \%$ de adición, en este punto la diferencia es del 3\%. En el otro extremo está el $4 \%$ de adición, en el que aparece la máxima diferencia, un $50 \%$.

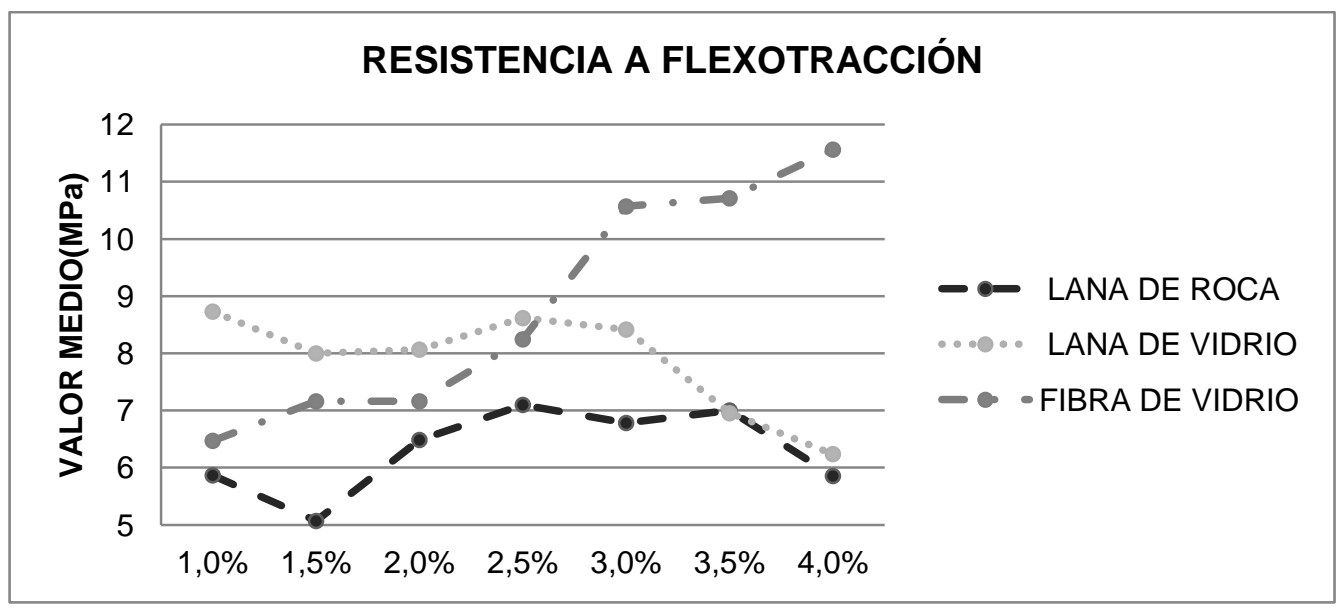

Fig. 2 "Comparativa Resistencia a flexotracción"

Los valores de resistencia a flexotracción de las series aditivadas con lana de vidrio son superiores a las aditivadas con fibra de vidrio para los porcentajes de adición inferiores al $3 \%$, obteniendo para el $1 \%$ de adición un valor un $26 \%$ superior. A partir del $3 \%$ de adición los valores de las series aditivadas con lana de vidrio caen mientras las aditivadas con fibra de vidrio aumentan llegando a su máxima diferencia, un $45 \%$, en el $4 \%$ de adición.

\section{3.- Resistencia a compresión.}

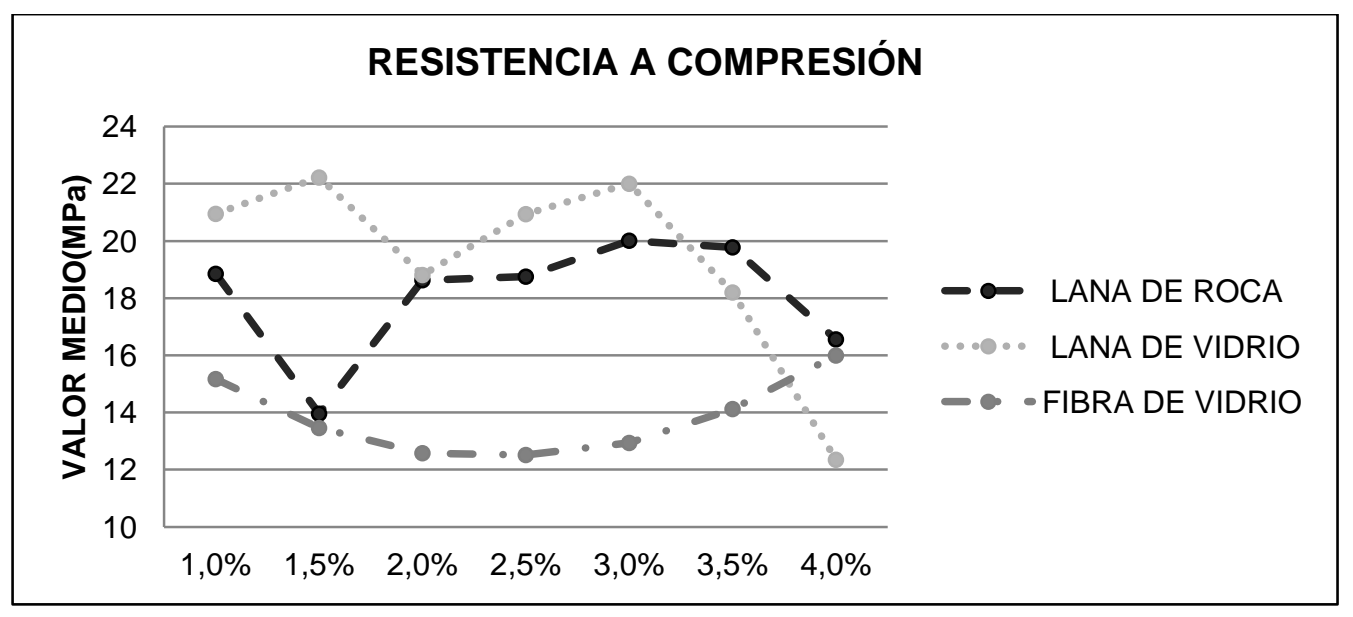

Fig. 3 "Comparativa Resistencia a compresión"

Las series aditivadas con fibra de vidrio obtienen una menor resistencia a compresión que las aditivadas con lanas minerales para todos los porcentajes de adición. En el caso de la lana de roca la mayor diferencia aparece para el 3\% de 
adición, en el que las series aditivadas con residuo de lana de roca superan en un $36 \%$ a las aditivadas con fibra de vidrio. Aparecen dos picos en el $1,5 \%$ y $4 \%$ de adición que deberán ser estudiados (fig. 3).

Los valores de resistencia a compresión también son inferiores para las series aditivadas con fibra de vidrio en comparación a las series aditivadas con residuo de lana de vidrio, encontrándose esta diferencia entre el 33\%, para un porcentaje de adición del $2 \%$, y el $41 \%$, para un porcentaje de adición del 3\%. A partir del 3\% de adición los valores descienden para las series aditivadas con lana de vidrio mientras aumentan para las aditivadas con fibra de vidrio llegando a ser estas últimas un $22 \%$ superiores, con el $4 \%$ de adición.

\section{6.- Conclusiones.}

A partir de los resultados se concluye que, con residuo de lana de roca se aumenta la Dureza Shore C y la resistencia a compresión en todos los porcentajes de adición estudiados. Sin embargo, los valores inferiores de resistencia a flexotracción son inferiores para todos sus porcentajes de adición.

Con respecto a las series aditivadas con residuo de lana de vidrio se concluye que mejoran la dureza superficial linealmente en torno al $5 \%$ para todos los porcentajes de adición, con respecto a las series aditivadas con fibra de vidrio. Los valores obtenidos en el ensayo de resistencia a compresión son mayores para todos los porcentajes de adición, excepto para el 4\%. Los valores de resistencia a flexotracción son superiores hasta el 3\% de adición.

Como conclusión final se establece que en general se obtienen mejores resultados con las series aditivadas con lanas minerales recicladas analizadas en este estudio que con las series aditivadas con las fibras utilizadas en la actualidad como refuerzo del yeso. Por tanto, la sustitución de la fibra de vidrio $\mathrm{E}$ por lanas minerales recicladas resulta viable.

\section{7.- Futuras líneas de investigación.}

Se proponen como futuras líneas de investigación, utilizar mayores porcentajes de adición, estudio sobre otros formatos de adición de la lana mineral, trabajar con otros tipos de tejidos procedentes de los RCD's u otros residuos industriales y mezclas mixtas entre diferentes tipos de fibras.

\section{REFERENCIAS}

[1] P. Villoria Sáez; M. Del Río Merino; C. Porras-Amores "Estimation of construction and demolition waste volume generation in new residential buildings in Spain". Waste Management \& Research 2012 Feb;30(2):137-46. Doi: 10.1177/0734242X11423955.

[2] Ministerio de Medio Ambiente y Medio Rural y Marino, 2008

[3] Real Decreto 105/2008, de 1 de febrero, por el que se regula la producción y gestión de los residuos de construcción y demolición. (1 de febrero de 2008).

[4] Rockwool firesafe insulation. (s.f.). Recuperado el Enero de 2013, de http://www. rockwool.es

[5] Isover. Saint-Gobain. (s.f.). Recuperado el Enero de 2013, de http://www.isover.es

[6] García Santos, A. (enero/febrero/marzo 2004. ). Aplicaciones constructivas de un material compuesto de escayola y fibras naturales de Typha Latifolia. Materiales de 
Construcción. CSIC. Incluida en el JCR del ISI. , ISSN 0465-2746, Vol. 54. № 273. Págs. 7377.

[7] Haselein, R., \& otros. (2002). Fabricaçao de chapas de partículas aglomeradas usando gesso como material cimentante. Ciência Forestal, V.12, n.1, 81-87.

[8] Khedari, J., \& otros. (2001). New lighweight composite constructions materials with low thermal conductivity. Cement \& Concrete Composities, Elsevier Ltd.27: 41-47.

[9] Oteiza San José, I. (s.f.). Estudio del comportamiento de la escayola reforzada con fibras de sisal, para componentes en viviendas de bajo coste. Tesis Doctoral.

[10] Santos, A. G. (s.f.). Modelo teórico del comportamiento mecánico del yeso y sus compuestos fibrosos poliméricos. Tesis Doctoral.

[11] del Rio Merino, M. (s.f.). Elaboración y aplicaciones constructivas de paneles prefabricados de escayola aligerada y reforzada con fibras de vidrio $\mathrm{E}$ y otros aditivos. Tesis Doctoral.

[12] del Río Merino, M., \& Comino Almenara, P. (2002). Análisis de los refuerzos mixtos de fibras de vidrio $E$ y fibras AR en la escayola, como alternativa a los refuerzos monofibras (homogéneos)". Materiales de Construcción, Vol. 52, no 268, pp. 33-42.

[13] Aguilar, J. C., Mendoza, D. N., Fuertes, R. H., González, B. B., Gilmore, A. T., \& Ramírez, R. P. (octubre-diciembre 2007). Caracterización del hormigón elaborado con áridos reciclados producto de la demolición de estructuras de hormigón. Materiales de Construcción, Vol. 57, 288, 5-15.

[14] Gónzalez Fonteboa, B., \& Martínez Abella, F. (2005). Hormigones con áridos reciclados: estudio de propiedades de los áridos y de las mezclas. Materiales de construcción , Vol 55, ำ 279.

[15] Madariaga, F. J., \& Macia, J. L. (enero-marzo 2008). Mezclas de residuos de poliestireno expandido (EPS) conglomerados con yeso o escayola para su uso en la construcción. Informes de la Construcción, Vol. 60, 509, 35-43.

[16] Andreloa, F., Barbieri, L., \& Lancellotti, P. (2005). Reciclado de residuos industriales en la fabricación de ladrillos de construcción. Materiales de Construcción, Vol 55, № 280.

[17] Sabador, E., Frías, M., Rojas, M. I., Vigil, R., García, R., \& José, J. T. (enero-marzo 2007). Caracterización y transformación de un residuo industrial (lodo de papel estucado) en un material con propiedades puzolánicas. Materiales de Construcción, Vol. 57, 285, 45-59.

[18] del Rio Merino, M., González Cortina, M., Izquierdo Gracia, P., Santa Cruz Astorqui, J., \& Salto weiss Azevedo, I. (s.f.). Los nuevos materiales de construcción como alternativa al reciclaje de los residuos industriales: mortero de cemento-caucho reciclado (ccr).

[19] del Río Merino, M. (2002). Patente no ES2170612A1, Yeso aligerado con corcho y su aplicación en paneles para construcción. . España, Madrid: OEPM. 